\title{
INCORPORANDO LA MULTIFUNCIONALIDAD EN LA EVALUACIÓN ECONÓMICA DE PROYECTOS DE RESTAURACIÓN DE BOSQUES NATIVOS SIEMPREVERDES EN EL SUR DE CHILE.
}

\author{
Schlegel, Bastienne ${ }^{2}$; Little, Christian ${ }^{1,2}$; Urrutia, Matías ${ }^{3}$; \\ Hernández, Germán ${ }^{3}$ y Pasten, Roberto ${ }^{3}$.
}

\section{RESUMEN}

Se evaluó la factibilidad económica de implementar iniciativas de restauración de bosques del Tipo Forestal Siempreverde, incorporando un análisis de costos y beneficios asociados a la ejecución de las actividades de manejo forestal y los retornos económicos potenciales por la comercialización de madera y de los servicios ecosistémicos secuestro de carbono y producción de miel.

Utilizando los métodos de transferencia de beneficios, análisis de rentabilidad a tasas de descuento diferenciadas y de sensibilidad económica, se evaluaron ocho escenarios que toman como referencia una serie de supuestos asociados a las tasas de crecimiento de los bosques en un plazo de 40 años, precios ajustados de los mercados de la madera, carbono (mercado internacional y nacional), miel y la posibilidad de acceder a un subsidio proporcionado por el Estado para recuperar bosques nativos (Ley 20.283).

Los resultados demuestran la compatibilidad e interdependencias entre la naturaleza ecológica de los bosques y las exigencias directas de los mercados, dando cuenta de la necesidad de incorporar el valor económico de los servicios ecosistémicos en el desarrollo de proyectos de restauración.

Este resultado es novedoso ya que entrega antecedentes que demuestran la importancia de considerar el valor económico de los bienes y servicios ecosistémicos de manera conjunta, lo que permite cambiar y expandir la racionalidad del uso de los bosques como un recurso renovable en relación a la escala temporal asociada al crecimiento de los bosques nativos.

Ello representa una oportunidad para incorporar dicho valor en políticas públicas, o las decisiones del sector privado, que permitan manejar los bosques con técnicas ecológicamente sólidas, dirigidas hacia la obtención de bosques multifuncionales que sustenten la provisión conjunta de bienes y servicios.

Palabras clave: Evaluación económica, Servicios ecosistémicos, Restauración, Multifuncionalidad.

\footnotetext{
${ }^{2}$ Instituto Forestal, Chile. Sede Los Ríos. bschlegel@infor.cl

${ }^{2}$ Center for Climate and Resilience Research (CR)2, Santiago, Chile.

${ }^{3}$ Instituto de Economía de la Facultad de Ciencias Económicas y Administrativas de la Universidad Austral de Chile.
} 


\section{SUMMARY}

The economic feasibility of implementing forest restoration initiatives in the evergreen forest type was evaluated, incorporating a cost benefits analysis associated to the implementation of forest management activities and expected benefits of the provision of timber and potential markets for the ecosystem services carbon sequestration and honey production.

Using the methods of benefit transfers, net revenue value, and sensibility to parameter changes, eight different scenarios were evaluated, considering a set of assumptions associated to rates of forest growth in a 40-year rotation, timber prices, carbon markets (domestic and international), honey-bee market, and the possibility to access to state subsidies available for native forest management (Law 20.283).

The results demonstrate the compatibility and interdependencies between the ecological nature of the forests and the direct demands of the markets, realizing the need to incorporate the economic value of ecosystem services in the development of restoration projects.

This result is novel since it provides background that demonstrates the importance of considering the economic value of ecosystem goods and services together, which allows changing and expanding the rationality of the use of forests as a renewable resource in relation to the time scale associated with native forest growth. This represents an opportunity to incorporate this value into public policies, or into the decisions of the private sector, that allow forests to be managed with ecologically sound techniques, aimed at obtaining multifunctional forests that support the combined provision of goods and services.

Key words: Economic valuation, Ecosystem services, Restauration, Multifunctionality. 


\section{INTRODUCCIÓN}

Los ecosistemas proveen una amplia variedad de bienes y servicios que satisfacen las necesidades del ser humano, como respirar aire puro, consumir alimentos, agua, recreación, realización espiritual, entre otros (Costanza et al., 1997, Figueroa y Pasten, 2015).

En ese sentido, los ecosistemas forestales dominados por bosques nativos en Chile proveen bienes, como aquellos productos derivados de la madera (ej. trozas aserrables, leña, otros), hongos y frutos; y también servicios, como la provisión de agua en calidad y cantidad, regulación microclimática, belleza escénica y otros (Lara et al., 2003; Little y Lara, 2014).

En las últimas décadas se ha propuesto que la valoración económica de servicios ecosistémicos sería una herramienta que permite alinear los objetivos de producción con los de conservación, al considerar simultáneamente el consumo de bienes con la mantención de la biodiversidad (Nahuelhual y Núñez, 2010).

Como respuesta, han surgido técnicas que ayudan a calcular el valor económico de los servicios ecosistémicos, las cuales reflejan la disposición que tienen las personas al cuidado de los ecosistemas en un contexto de escasez. Figueroa y Pasten (2015) detallan que, existiendo una disposición a sacrificar un recurso por otro, entonces se le puede otorgar un valor económico a estos.

En Chile, la valoración económica de servicios ecosistémicos asociado a los bosques nativos ha sido una forma de reflejar el beneficio adicional que estos tienen para la producción de bienes que comúnmente se transan en el mercado, como por ejemplo los derivados de la madera.

Experiencias de valoración económica señalan algunas cifras anuales entre 61,2 y 162 US\$/ha para bosques nativos presentes en cuencas forestales vinculadas a la provisión de agua (Núñez et al. 2006), valores que según métodos de cálculo podrían ser $400 \%$ superiores (Figueroa y Pastén, 2008); 1,6-6,3 US\$/ha por la belleza escénica de los bosques presentes en Parques Nacionales (Nahuelhual et al., 2007), 35-178 US\$/ha cuando se considera solo el área de uso intensivo y 26,3 US $\$$ /ha como resultado de la protección de los bosques ante la pérdida de suelo (Oyarzún y Huber, 1997).

Si bien los servicios ecosistémicos han sido reconocidos en instrumentos de política forestal en Chile, dichos instrumentos son insuficientes para corregir distorsiones de mercado a favor del manejo y conservación de los bosques ${ }^{3}$.

De hecho, en el caso de la Ley del Bosque Nativo (Ley $\mathrm{N}^{\circ} 20.283,2008$ ), pese a incluir una definición de "servicios ambientales", como "aquellos que brindan los bosques nativos que inciden directamente en la protección y mejoramiento del medio ambiente", no existen bonificaciones que incluyan actividades tendientes a mantener o aumentar tales servicios (Lara et al., 2010).

En Chile, alrededor de $70 \%$ de los bosques nativos son de propiedad privada (Lara et al., 2011), por lo tanto, las decisiones sobre su conservación y manejo consideran una perspectiva socioeconómica asociada a los beneficios directos $e$ indirectos que estos les proveen (Droppelmann et al., 2019).

\footnotetext{
${ }^{3}$ Pastén et al. (2018) muestran los casos en que los servicios ecosistemicos tienen reconocimiento legal en Chile.
} 
Lo anterior, se ve reflejado en paradigmas reduccionistas que consideran a los bosques como áreas exclusivas destinadas a la preservación de la naturaleza y biodiversidad, donde el propietario asume un costo de oportunidad, o una visión de estos como áreas netamente productoras de madera o de crianza de animales, caso en el que el propietario percibe beneficios económicos tangibles.

Esta última perspectiva, que ha regido la historia de uso de los bosques en Chile, ha dejado miles de hectáreas en un estado de conservación con funciones ecológicas limitadas, disminuyendo la oferta de bienes y servicios (Vergara et al., 2019).

Por lo anterior, es clave que el Estado juegue un rol catalizador en el desarrollo de mercados que permitan evaluar proyectos de manejo forestal con enfoques multifuncionales, los cuales realizan la habilidad de estos ecosistemas de proveer simultáneamente múltiples bienes y servicios que se derivan de sus funciones y procesos (Manning et al., 2018).

Considerando que la restauración ha sido propuesta como una de las estrategias de manejo que permitirían recuperar la productividad natural de los sitios y la provisión de bienes y servicios ecosistémicos derivados de los bosques nativos (Little y Lara, 2014), el objetivo de este trabajo es analizar la factibilidad económica de ejecutar iniciativas de restauración, incorporando para dicho análisis un enfoque multifuncional del Valor Económico Total (VET) de los beneficios asociados al bosque, como proveedores de madera y de servicios ecosistémicos como secuestro de carbono y producción de miel.

\section{MÉTODO}

\section{Caso de Estudio}

Se trabajó con la información proveniente de tres ensayos de restauración de bosques nativos establecidos en el año 2016, cuyo propósito es la recuperación de bienes derivados de la madera y los servicios ecosistémicos secuestro de carbono y producción de miel.

Los ensayos se encuentran localizados en la región de Los Lagos (Figura N¹a), específicamente en los sectores de Butalcura, Puntra y Pargua, y se caracterizan por presentar bosques secundarios del tipo forestal siempreverde con una composición mixta de las especies canelo, ulmo, tepa, coigüe (Drimys winteri, Eucryphia cordifolia, Laureliopsis philippiana y Nothofagus dombeyi) y Mirtaceas, como luma y melí (Amomyrtus luma y Amomyrtus meli).

Estos bosques presentan una densidad entre 1.240 y 1.600 árboles/ha, áreas basales de 12,7 a $41,1 \mathrm{~m}^{2} /$ ha y una estructura (distribución de los árboles, sus copas, estratificación vertical, etc.) que es el resultado de una cosecha de árboles sin criterios silvícolas (floreo) y el uso del bosque como un área para el pastoreo de animales domésticos.

Estos bosques comúnmente han sido denominados como "degradados", cuya característica es la gran abundancia de especies de sotobosque (ej. Quila, Chusquea quila) que inhibe la regeneración natural de las especies arbóreas (Vergara et al., 2019).

Cada ensayo fue cercado a fin de evitar el ingreso de animales de crianza y se realizaron diversas actividades silvícolas siguiendo una técnica de plantación suplementaria en núcleos bajo dosel (Saha et al., 2016), la cual consiste en la eliminación de especies competidoras a la regeneración en pequeños sectores del bosque $\left(\sim 15 \mathrm{~m}^{2}\right)$ y habilitación de micro-sitios para el 
establecimiento de las especies arbóreas de interés, ya sea a través de regeneración natural o artificial (Figura $\mathrm{N}^{\circ} 1 \mathrm{~b}$ ).

En cada ensayo se establecieron 80 núcleos por hectárea, en los cuales se plantaron 15 individuos de las especies ulmo o avellano (Gevuina avellana), lográndose una densidad final de plantación de 1.200 árboles por hectárea (Figura $N^{\circ} 1 \mathrm{c}$ ).

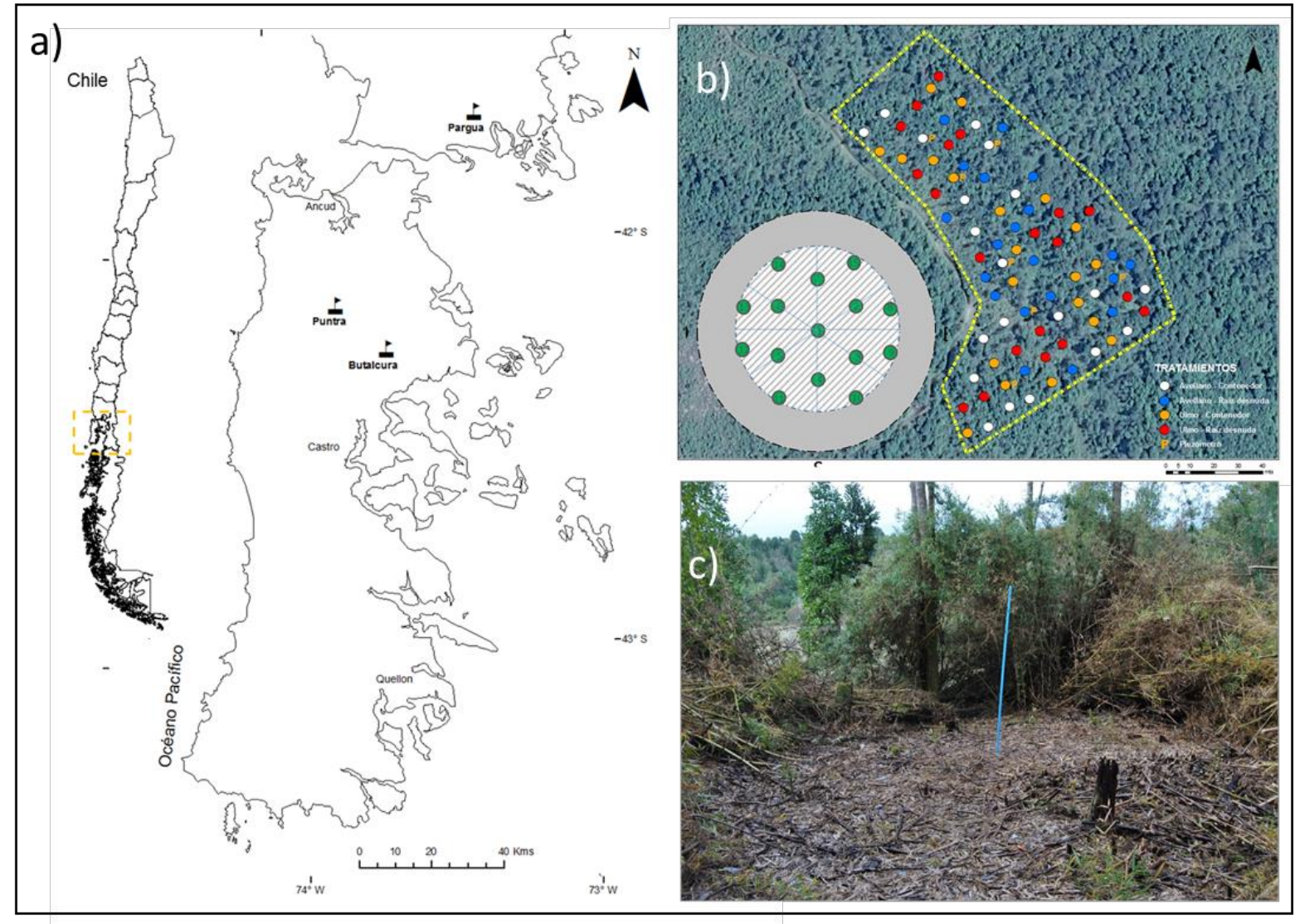

Figura $N^{\circ} 1$

UBICACIÓN DE LOS ENSAYOS EN BUTALCURA, PUNTRA Y PARGUA (a). ESQUEMA DE PLANTACIÓN SUPLEMENTARIA EN NÚCLEO CON LAS ESPECIES ULMO Y AVELLANO A RAÍZ CUBIERTA Y DESNUDA (b). EJEMPLO DE DISPOSICIÓN DE NÚCLEOS EN TERRENO (c).

\section{Valoración Económica de la Restauración}

Para estimar el valor económico total (VET) se utilizó el método de transferencia de beneficios (TB) con una proyección de 40 años a partir del 2020. Particularmente, se utilizó la transferencia del valor unitario y la función de beneficios (Figueroa y Pastén, 2011) que utiliza información de costos y beneficios a partir de fuentes directas asociadas al desarrollo de los ensayos de restauración o de sitios con similares características, cuyo principal objetivo sea asegurar la regeneración del bosque. 
Respecto de los costos de la restauración, estos fueron determinados a partir del valor real promedio observado en los tres ensayos (Cuadro $\mathrm{N}^{\circ} 1$ ). Las cuatro primeras partidas son asumidas como acciones directas de manejo silvícola en el primer año del proyecto, mientras que el control de la vegetación competidora en los años 1 y 3 , y el cuidado y vigilancia como una anualidad.

\section{Cuadro $\mathrm{N}^{\circ} 1$}

\section{COSTOS ASOCIADOS A LAS ACTIVIDADES DE RESTAURACIÓN}

\begin{tabular}{|l|c|l|}
\hline Actividad & $\begin{array}{c}\text { Costo } \\
\text { Total } \\
\text { (US\$/ha) }\end{array}$ & \multicolumn{1}{|c|}{ Detalle } \\
\hline Cercado & 493,50 & $\begin{array}{l}\text { Costo real por cercado con malla o alambre de 4 } \\
\text { hebras. Valores incluyen la compra de materiales y } \\
\text { mano de obra para la instalación del cerco en el } \\
\text { perímetro del ensayo. }\end{array}$ \\
\hline $\begin{array}{l}\text { Eliminación vegetación } \\
\text { competidora }\end{array}$ & $1.209,64$ & $\begin{array}{l}\text { Costos reales por ejecución de la faena de roce de } \\
\text { núcleos por hectárea dispuestos en forma sistemática. }\end{array}$ \\
\hline Plantas & $1.027,08$ & $\begin{array}{l}\text { Compra de plantas en vivero y traslado al lugar del } \\
\text { ensayo. Se consideraron plantas de 1 año, con } \\
\text { valores por planta de US\$ 0,86 para Euchryphia } \\
\text { cordifolia (Ulmo) a raíz cubierta y US\$1,17 para ulmo } \\
\text { a raíz desnuda, y valores de US\$ 0,93 de Gevuina } \\
\text { avellana (Avellano) a raíz cubierta y raíz desnuda. }\end{array}$ \\
\hline $\begin{array}{l}\text { Actividad de plantación } \\
\text { en núcleos }\end{array}$ & 235,39 & $\begin{array}{l}\text { Costos reales por ejecución de faena de plantación } \\
\text { vía contratación de personal calificado (contratista } \\
\text { forestal). }\end{array}$ \\
\hline $\begin{array}{l}\text { Control de vegetación } \\
\text { competidora }\end{array}$ & 604,82 & $\begin{array}{l}\text { Costos reales por ejecución de faena vía contratación } \\
\text { de personal calificado (contratista forestal). }\end{array}$ \\
\hline Mantención anual & 196,16 & $\begin{array}{l}\text { Supuesto de anualidad por mantención de cercos y } \\
\text { vigilancia. }\end{array}$ \\
\hline
\end{tabular}

Dado el carácter privado de los bosques, se calculó el potencial beneficio económico por producción de madera, como un producto al término del horizonte de evaluación del proyecto, e ingresos anuales por secuestro de carbono y producción de miel.

Para el caso de la madera, se tomó como supuesto una extracción de $40-80 \mathrm{~m}^{3} / \mathrm{ha}$ a través de una corta de selección que proyecta el bosque hacia una estructura multietánea. Esto correspondería a un tercio del crecimiento acumulado durante 40 años a una tasa promedio de 4-6 $\mathrm{m}^{3}$ /ha/año.

\footnotetext{
${ }^{4}$ Se consideró un valor de dólar de 764,69 \$/US\$ con fecha 11 de enero de 2020, (Banco Central, 2020a).
} 
Del volumen cosechado, se asume que un $90 \%$ equivale a leña y un $10 \%$ a trozas aserrables (Donoso y Pilquinao, 2013). Respecto de la captura anual de $\mathrm{CO}_{2}$, se calculó tomando como antecedente el mismo incremento en volumen del bosque y una serie de datos paramétricos, como la densidad básica, factores de expansión a biomasa aérea y biomasa de raíces, y fracción de carbono (Eq. 1) (IPCC, 2006). Los valores y fuente de información para cada parámetro se detallan en el Cuadro $\mathrm{N}^{\circ} 2$.

$$
\text { Eq. } 1 \quad \operatorname{CO} 2 e=\sum\left(V_{j} * D_{j} * F E B_{j}\right) *\left(1+R_{j}\right) * F C * F C o n
$$

Cuadro $\mathrm{N}^{\circ} 2$

DATOS PARAMÉTRICOS PARA EL CÁLCULO DE LA CAPTURA ANUAL DE $\mathrm{CO}_{2}$

\begin{tabular}{|l|c|l|}
\hline Parámetro & Valor & \multicolumn{1}{|c|}{ Fuente } \\
\hline $\begin{array}{l}\text { V: Incremento en volumen } \\
\text { comercial }\left(\mathrm{m}^{3} / \mathrm{ha} / \mathrm{año}\right)\end{array}$ & $4-6$ & $\begin{array}{l}\text { (INFOR, 2018). Estimación a partir del Inventario } \\
\text { Forestal Nacional para el TF Siempreverde. }\end{array}$ \\
\hline D: Densidad básica (t/m ${ }^{3}$ ) & 0,496 & $\begin{array}{l}\text { (Gayoso et al., 2002; Pérez, 1983 y Hernández y } \\
\text { Pinilla, 2010). Calculado como promedio a partir de } \\
\text { los valores para canelo, ulmo, avellano, tepa, } \\
\text { coigüe, luma, tineo y trevo. }\end{array}$ \\
\hline $\begin{array}{l}\text { FEB: Factor de expansión a } \\
\text { biomasa aérea }\end{array}$ & 1,75 & $\begin{array}{l}\text { (Gayoso et al., 2002). Calculado como promedio a } \\
\text { partir de especies nativas. }\end{array}$ \\
\hline $\begin{array}{l}\text { R: Proporción de raíces en } \\
\text { biomasa aérea }\end{array}$ & 0,29 & $\begin{array}{l}\text { (Gayoso et al., 2002). Calculado como promedio a } \\
\text { partir de especies nativas. }\end{array}$ \\
\hline FC: Fracción de carbono & 0,47 & (Gayoso et al., 2002) \\
\hline $\begin{array}{l}\text { Fcon: Factor de conversión } \\
\text { C/CO }\end{array}$ & 3,667 & (IPCC, 2006) \\
\hline
\end{tabular}

Los costos e ingresos fueron proyectados a 40 años considerando un incremento de los valores asociado a la variación de Índice de Precios al Consumidor (IPC), utilizando como medida de inflación el valor promedio en los últimos 10 años observado en Chile (2,98\%) (linflation.eu, 2020).

Para estimar el VET (Eq. 2) (Ninan y Kontoleon, 2016) proveniente de la madera se asumieron costos de producción e ingresos similares a los proporcionados por Donoso y Pilquinao (2013) para ensayos de silvicultura en bosques siempreverdes adultos.

Los valores fueron de 37,4 y $34,5 \mathrm{US} \$ / \mathrm{m}^{3}$ para los costos de producción de leña y trozas aserrables y de 85,4 y $138 \mathrm{US} \$ / \mathrm{m}^{3}$ para los ingresos, respectivamente.

Para la captura de $\mathrm{CO}_{2}$ se utilizó una proyección lineal del incremento en biomasa, estimándose una captura anual entre 7,7 y $11,6 \mathrm{tCO}_{2} /$ ha. A esta captura se descontó una emisión de $2 \mathrm{tCO}_{2}$, derivada de la eliminación de la vegetación competidora, principalmente quila, al inicio 
del proyecto. Lo anterior se calculó a partir del dato de acumulación de 17,66 t/ha de biomasa de quila (De la Fuente y Pacheco, 2017) y la superficie intervenida en los núcleos de plantación.

A la captura y emisión fueron asociadas dos fuentes de precio por tonelada de $\mathrm{CO}_{2}$ capturada, correspondiente al observado en el mercado internacional de 42 US\$ (EPA, 2016) y el nacional de 5 US\$ (Ley N²0.780, 2014).

El valor internacional fue ajustado considerando la transferencia de valor del dólar al mercado nacional $\left(D A P_{1}\right)$ (Eq. 3) (Figueroa y Pastén, 2011), llegando a un valor de 17,24 US\$. Para ello se tomaron como referencia los ingresos per cápita de 55.719 US\$ para Estados Unidos y 22.874 US\$ para Chile, y valores de producto interno bruto (PIB) en dólares a precios constantes del año 2011 (World Bank, 2019a).

Cuadro $\mathrm{N}^{\circ} 3$

ECUACIONES PARA EL ANÁLISIS ECONÓMICO

$$
\begin{aligned}
& \text { (Eq. 2) } V E C C=P * C \\
& \text { (Eq. 3) } D A P_{1}=D A P_{0} *\left(\frac{P I B_{1}}{P_{1} B_{0}}\right)^{\varepsilon} \\
& \text { (Eq. 4) } V E P=P M * N C \\
& \text { (Eq. 5) } V A N=\sum_{j=0}^{f} \frac{B_{j}-C_{j}}{(1+i)^{r}} \\
& \text { (Eq. 6) } T I R=i ; \sum_{j=0}^{f} \frac{B_{j}-C_{j}}{(1+i)^{r}}=0 \\
& \text { (Eq. 7) } P R I=a+\frac{b}{d}
\end{aligned}
$$

(2) VECC: Valor Económico de la Captura de Carbono

P: Precio anual de $\mathrm{CO}_{2}$

C: Captura anual de $\mathrm{CO}_{2}$

(3) DAP: disposición a pagar por reducción de emisiones de $\mathrm{CO}_{2}$ (precio $\mathrm{CO}_{2}$ )

$P I B_{0}$ : nivel de ingreso per cápita en términos de paridad de poder adquisitivo de Estados Unidos $P I B_{1}$ : nivel de ingreso per cápita en términos de paridad de poder adquisitivo de Chile.

$\varepsilon$ : Elasticidad ingreso de la disposición a pagar marginal (en algunos estudios se asume $\varepsilon=1$ )

(4) VEP: Valor económico del servicio de producción de miel

PM: Precio de la miel

$N C$ : Número de colmenas

(5) VAN: Valor actual neto

$B$ : Beneficio en el año 'j'

$C_{j}$ : Costo en el año 'j'

$r$. Años del proyecto

i: Tasa de descuento

(6) TIR: Tasa interna de retorno

$B_{j}$ : Beneficio en el año 'j'

$C_{j}$ : Costo en el año 'j'

r. Años del proyecto

$i$ : Tasa de descuento

(7) PRI: Período de recuperación de la inversión

a: número de período inmediatamente anterior hasta recuperar la inversión

b: suma de los flujos descontados hasta el período 'a' (incluye ingresos y egresos)

d: valor del flujo descontado en que se recupera la inversión (flujo del período a+1). 
Para el beneficio económico asociado al secuestro de carbono se determinó una proyección de valores de los mercados propuestos, considerando como fuente la variación de la tasa del crecimiento del PIB per cápita observada en Chile en los últimos 10 años $(1,96 \%)$ World Bank, 2019b). Para el precio del mercado internacional (EPA, 2016) dicha proyección se realizó a partir del año 2020, mientras que para el mercado nacional a partir del año 2026 (Ley $\mathrm{N}^{\circ} 20.780$, 2014). La proyección de precios es un ajuste necesario dado el potencial cambio en la disposición a pagar para reducir los niveles de emisión de carbono según el nivel de ingresos de los consumidores (Figueroa y Pastén, 2011).

Para el caso de la producción de miel se estimó el monto en dinero que recibiría un propietario de bosque nativo por la producción de miel (Eq. 4), considerando que 1 ha de bosque es capaz de sostener entre 10 a 20 colmenas, siendo lo más común 12 , y que el propietario recibiría aproximadamente 1 kilo de miel por cada colmena instalada o su equivalente en dinero al precio de mercado.

Para el cálculo se realizó una consulta a productores locales de miel quienes entregan información respecto a la trashumancia de abejas y niveles de producción. Se estableció \$6,54 US\$/kg como valor de mercado interno de la miel ( $P$. Cáceres ${ }^{5}$, comunicación personal, 11 de diciembre de 2019). De la misma forma que para el precio de la madera, se realizó un ajuste de precios tomando la variación de Índice de Precios al Consumidor (IPC, 2,98\%) y se asume un contrato anual por trashumancia para los 40 años del proyecto.

\section{Evaluación de Escenarios}

Se evaluaron 8 escenarios basados en el cruce de las variables que determinan la rentabilidad de los proyectos (Cuadro $\mathrm{N}^{\circ} 3$ ), vale decir; tasa de crecimiento del bosque, el precio del carbono en el mercado internacional y nacional, la tasa de descuento y la posibilidad de acceder a bonificaciones de actividades para la recuperación de bosques nativos (Ley $\mathrm{N}^{\circ} 20.283$, 2008) al segundo año del horizonte del proyecto.

Para el análisis se utilizaron tres herramientas asociadas a la evaluación de proyectos: a) El Valor Actual Neto (VAN) (Eq. 5) que entrega información sobre la factibilidad de aceptar o rechazar el proyecto basado en el flujo de ingresos y egresos (Mete, 2014); b) la Tasa Interna de Retorno (TIR) (Eq. 6), como el factor de descuento mínimo para ejecutar un proyecto (VAN $=0$ ) (Mete, 2014), y c) el Período de Recuperación de la Inversión descontado (PRI) (Eq. 7) que indica el tiempo necesario para recuperar los costos incurridos en la ejecución de las actividades (Mascareñas, 2008).

Respecto a la tasa de descuento, se utilizó una de $2,25 \%$ que corresponde a la tasa promedio de 3\% que utiliza EPA (2016) luego de aplicar el principio ecológico propuesto por Gollier (2010); y otra tasa de $5 \%$ que se asemejaría a un proyecto en el mercado financiero. Para la bonificación estatal se consideró la posibilidad o no de acceder a un máximo de 10 UTM (Unidad Tributaria Mensual) por hectárea para actividades silviculturales destinadas a manejar y recuperar bosques nativos con fines de producción maderera (Ley №20.283, 2008. Tabla de valores 2015 , www.conaf.cl).

Para cada escenario se buscó el valor del precio del $\mathrm{CO}_{2}$, la TIR y bonificación que resultan en un VAN igual a cero (ceteris paribus).

\footnotetext{
${ }^{5}$ Gerente General del Consorcio Apícola, Valdivia.
} 
Cuadro $\mathrm{N}^{\circ} 3$

ESCENARIOS DE RENTABILIDAD

\begin{tabular}{|l|c|c|c|c|c|c|c|c|}
\hline \multirow{2}{*}{ Variable } & \multicolumn{8}{|c|}{ Escenarios } \\
\cline { 2 - 9 } & $\mathbf{1}$ & $\mathbf{2}$ & $\mathbf{3}$ & $\mathbf{4}$ & $\mathbf{5}$ & $\mathbf{6}$ & $\mathbf{7}$ & $\mathbf{8}$ \\
\hline $\mathrm{CO}_{2}$ (USD\$/t) & \multicolumn{6}{|c|}{17,24} & \multicolumn{5}{c|}{5,00} \\
\hline Tasa de descuento (\%) & \multicolumn{3}{|c|}{2,25} & \multicolumn{3}{|c|}{6} & \multicolumn{3}{|c|}{2,25} & \multicolumn{3}{c|}{6} \\
\hline Bonificación $\left(\mathrm{UTM}^{6}\right)$ & 10 & 0 & 10 & 0 & 10 & 0 & 10 & 0 \\
\hline
\end{tabular}

Finalmente, utilizando una simulación de Montecarlo (Raychaudhuri, 2008) se realizó un análisis de sensibilidad para los escenarios 1 y 5 , los cuales únicamente cambian el valor del mercado de $\mathrm{CO}_{2}$, siendo las fuentes de variación: a) La tasa de crecimiento del bosque, b) EI PIB per cápita, c) EI IPC, d) El precio de la miel asociado al número de colmenas por hectárea.

Para las tres primeras variables se utilizó una distribución normal de los valores, mientras que para la miel una distribución triangular transformada, con 10 colmenas como valor inferior, 20 como superior y una moda de 12. Se generaron 100 valores aleatorios para cada una de las variables señaladas anteriormente dando cuenta de 100 valores del indicador VAN.

\section{RESULTADOS}

\section{Análisis de Rentabilidad}

Para los casos y supuestos estudiados se estimó que el bosque en restauración entregaría ingresos brutos equivalentes a $1.492,7$ y 2.239,1 US $\$$ por concepto de producción de madera para las tasas de crecimiento de 4 y $6 \mathrm{~m}^{3} /$ ha/años, respectivamente.

A dicho monto se sumaría $6.070,2$ US $\$$ por concepto de miel y $1.632,3-6.170,0$ US $\$$ y $3.162,8$ - 11.957,8 US\$, para los supuestos de tasas de crecimiento señalados anteriormente por los precios el carbono en los mercados nacionales e internacionales, respectivamente.

Considerando el flujo de costos-beneficios y los 8 escenarios evaluados, el VAN fluctuaría entre 4.181,5 US\$ y $-4.345,9$ US\$ (Figura $\mathrm{N}^{\circ} 2 \mathrm{a}$ ), y períodos de recuperación de la inversión entre 27 y más de 100 años (Figura $\mathrm{N}^{\circ} 2 \mathrm{~b}$ ).

\footnotetext{
${ }^{6}$ UTM de Ch \$ 49.673 (Banco Central, 2020b), consulta 12 de enero de 2020.
} 


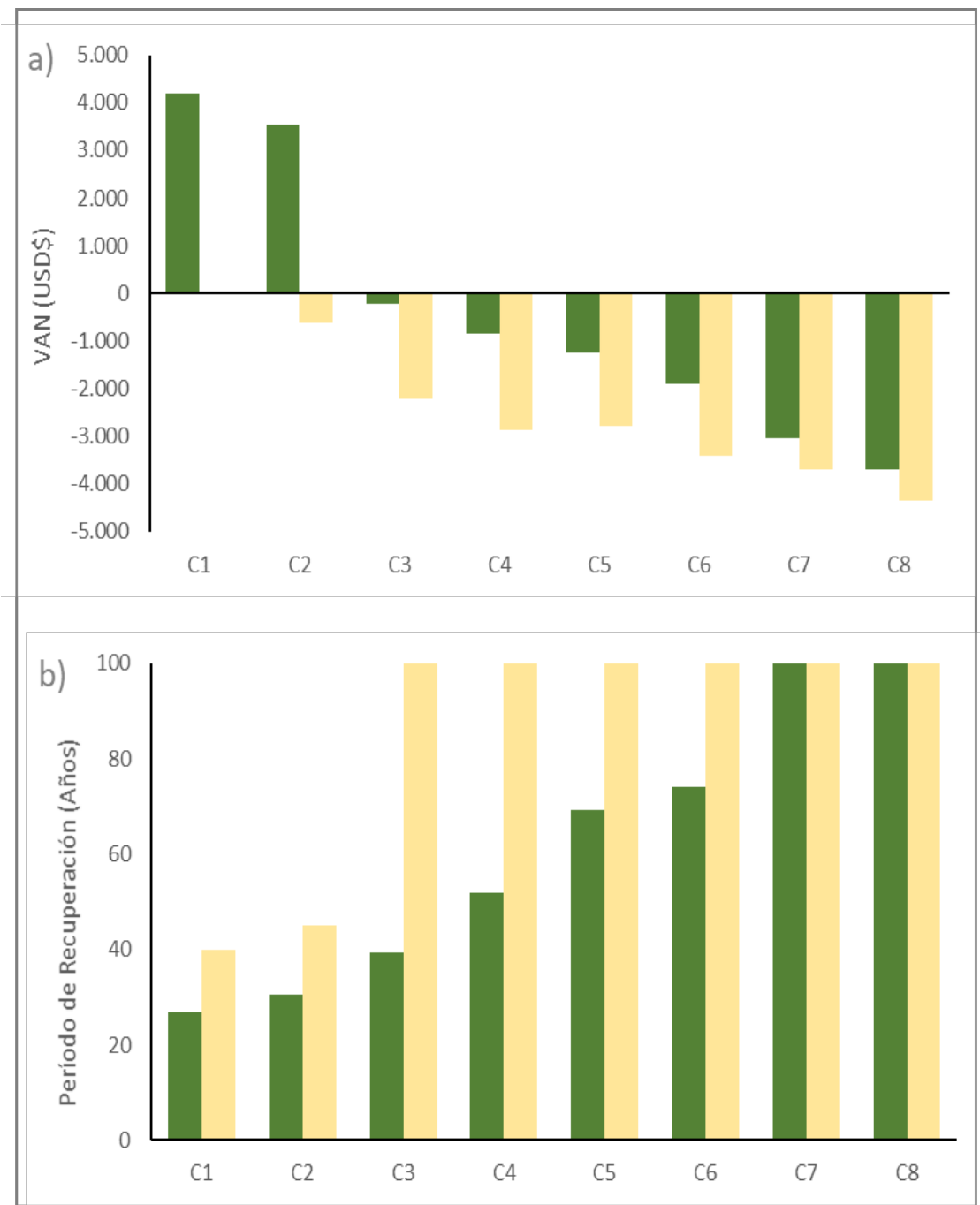

a) VAN y b) PRI. Barras negras y grises representan tasas de crecimiento de 6 y $4 \mathrm{~m}^{3} / \mathrm{ha} / \mathrm{año}$, respectivamente.

Figura $\mathrm{N}^{\circ} 2$

INDICADORES DE EVALUACIÓN DE PROYECTOS PARA LOS ESCENARIOS EVALUADOS

La progresión del VAN para las tasas de incremento volumétrico de 6 y $4 \mathrm{~m}^{3} / \mathrm{ha} / \mathrm{año}$ comienza a ser positiva (Figura $\mathrm{N}^{\circ} 3 \mathrm{a}$ ) a partir del año 3 y 4 para escenarios que incorporan el valor del $\mathrm{CO}_{2}$ de mercado internacional (A), mientras que para el mercado nacional (Figura $\mathrm{N}^{\circ} 3 \mathrm{~b}$ ) a partir de los años 17 y 24 , respectivamente (B).

Se observó que el escenario 3 presentó mayores valores que el escenario 2 durante los primeros 22 y 33 años, sin embargo, este último llega a resultados más favorables al final del período. Esto se produce por efecto de los costos de roce de la vegetación competidora al inicio del proyecto, la bonificación inicial y los niveles más bajos de la tasa de descuento. 


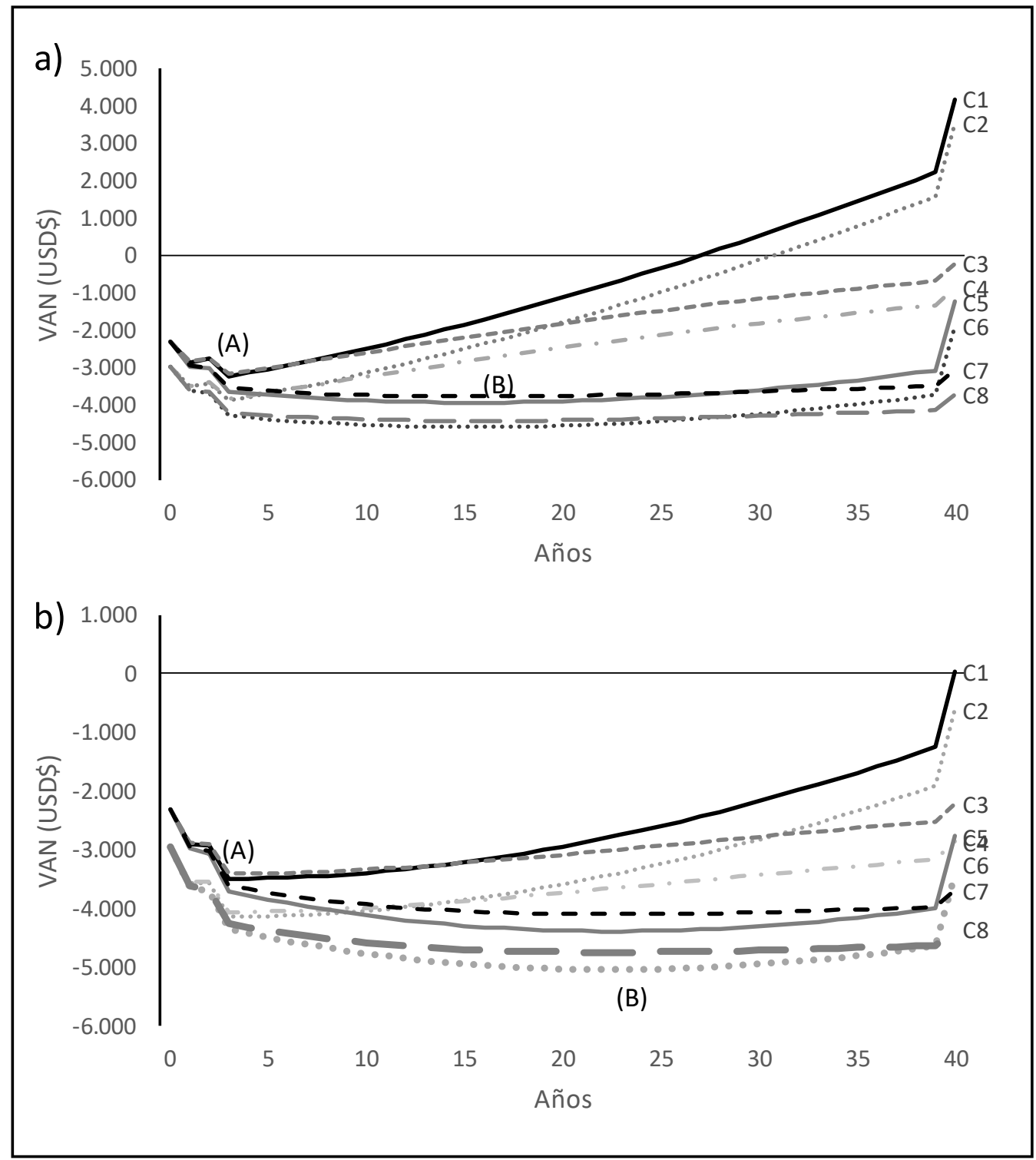

(A) y (B) representan el punto de inflexión de la tendencia.

Figura $\mathrm{N}^{\circ} 3$

VALOR ACTUAL NETO PROGRESIVO PARA LOS ESCENARIOS PROPUESTOS

En los escenarios con precio del $\mathrm{CO}_{2}$ internacional se observó que la TIR, que entrega un valor de VAN igual a cero, resulta ser levemente inferior a la tasa ecológica de $2,25 \%$ (Figura $\mathrm{N}^{\circ} 4 a$ ). En tanto, para obtener dicha tasa se requiere un precio mínimo de venta de 17,1 y 7,5 US $\$$ por tonelada capturada para tasas de crecimiento de 4 y $6 \mathrm{~m}^{3} / \mathrm{ha} / a n ̃ o$, respectivamente, valor que se incrementa en 3 y 1,5 US\$ si no se incluye la bonificación de 10 UTM para manejo de bosque nativo (Ley $\mathrm{N}^{\circ} 20.283$ ).

Para la tasa comercial de $6 \%$, el valor mínimo por tonelada de $\mathrm{CO}_{2}$ debiera ser de 36,28 y 18,2 US\$ (Figura $\mathrm{N}^{\circ} 4 \mathrm{~b}$ ) para proyectos con bonificación y tasas de crecimiento de 4 y 6 
$\mathrm{m}^{3} /$ ha/año, respectivamente. Así mismo, al mantener constante los precios de mercado de carbono y la tasa de descuento, el valor mínimo de fomento alcanza valores entre 624,2 US\$ y 4.345,9 US\$ para los casos más y menos favorables, relacionados con el precio del carbono, tasa de descuento comercial y crecimiento del bosque (Figura $\mathrm{N}^{\circ} 4 \mathrm{c}$ ).

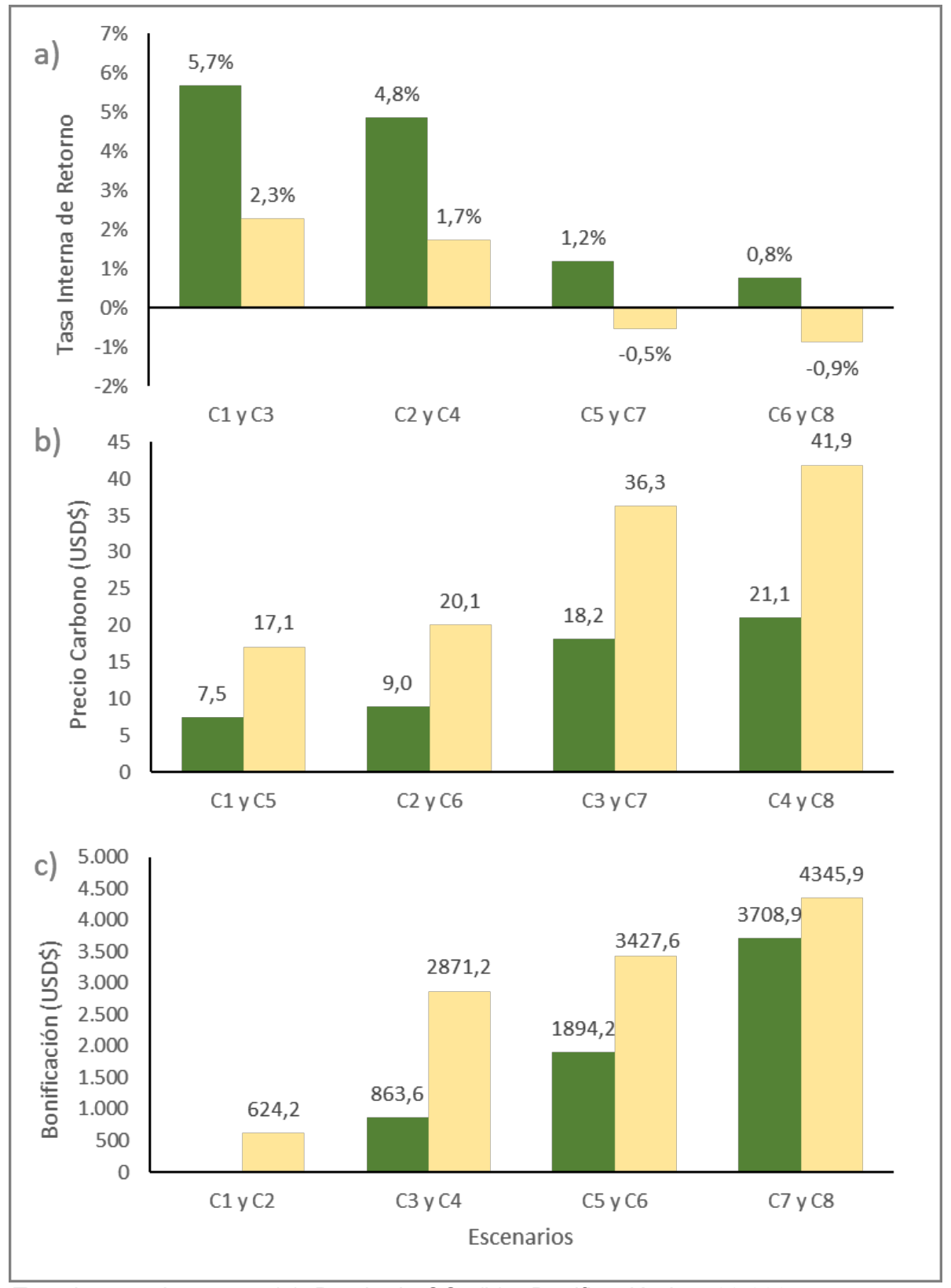

Tasa interna de retorno (a), Precio de $\mathrm{CO}_{2}$ (b) y Bonificación Ley 20.283

(c) Barras verdes y amarillas representan tasas de crecimiento 6 y $4 \mathrm{~m}^{3} /$ ha/año, respectivamente.

Figura $\mathrm{N}^{\circ} 4$

CÁLCULO DE VARIABLES QUE GENERAN VAN = 0 
Tomando los escenarios propuestos para el análisis de sensibilidad (1 y 5), se observó que la rentabilidad promedio asociada a los mercados nacionales e internacionales del carbono fueron de $-3.232,8$ y $1.119,1$ US $\$$, respectivamente. Para el primer escenario se observó una probabilidad de $80 \%$ de tener un resultado positivo, mientras que para la del mercado nacional esta fue nula.

Cabe señalar la importancia de los precios de la madera, producción de miel y $\mathrm{CO}_{2}$ en la contribución al VAN, donde el valor de estos muestran la importancia de los mercados. En el caso del precio internacional de $\mathrm{CO}_{2}$, este mercado representa más de la mitad del VAN (55\%), cifra que disminuye a menos de la mitad con el precio del mercado nacional, llegando a ser el valor de la madera y de la miel las principales fuentes de ingreso del proyecto, con 39 y $37 \%$, respectivamente (Figura $\mathrm{N}^{\circ} 5$ ).

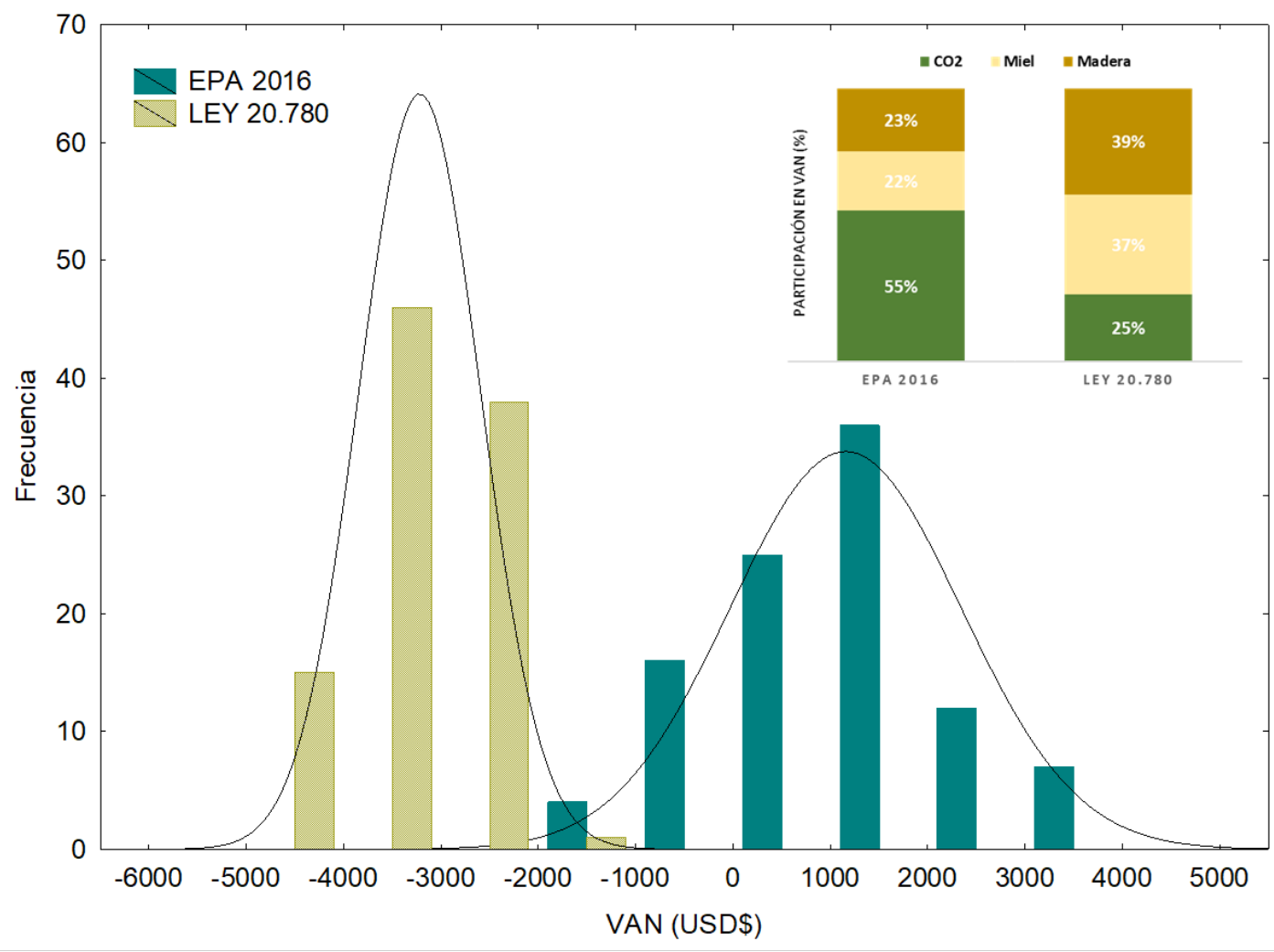

\section{Figura $\mathrm{N}^{\circ} 5$ \\ ANÁLISIS DE SENSIBILIDAD PARA LA RENTABILIDAD DE LOS PROYECTOS DE RESTAURACIÓN EN LOS MERCADOS NACIONALES E INTERNACIONALES DE CARBONO Y PARTICIPACIÓN RELATIVA DE LOS MERCADOS DE LA MADERA, PRODUCCIÓN DE MIEL Y $\mathrm{CO}_{2}$ EN EL VAN}

\section{DISCUSIÓN}

Los ensayos de restauración analizados en este trabajo corresponden a experiencias desarrolladas en sitios de baja productividad, siendo una característica de los bosques siempreverdes sometidos a entresaca selectiva de árboles (floreo) y el uso de estos bosques como áreas de pastoreo y resguardo para la crianza de animales. Estas acciones han sido identificadas 
como agentes de degradación que inhiben la regeneración natural de los bosques y la productividad del sitio (Vergara et al., 2019).

Considerando que en estos sitios la rentabilidad de la producción maderera puede ser baja, un enfoque económico que capture las múltiples funciones de los bosques podría representar una oportunidad para incorporar valores intangibles al mercado y ofrecer un beneficio a los propietarios. Para los ensayos analizados, se determinó que la inversión necesaria para asistir la recuperación del bosque (restauración) alcanzó valores cercanos a los $3.700 \mathrm{US} \$ / \mathrm{ha}$. Teniendo en cuenta el flujo de costos y beneficios asociados a la madera y los servicios ecosistémicos analizados (secuestro de $\mathrm{C}$ y producción de miel), únicamente dos de los escenarios entregaron resultados positivos respecto a la factibilidad económica de ejecutar el proyecto. La productividad del bosque, el precio del $\mathrm{CO}_{2}$ en los mercados (internacional y nacional) y la tasa interna de retorno, resultan claves para recuperar la inversión inicial y capturar adecuadamente el valor de los servicios ecosistémicos.

Para el horizonte de 40 años proyectados, el precio del carbono a 5 US\$/t entregó cifras negativas de VAN, independiente de la tasa de descuento o la posibilidad de recibir una bonificación del Estado para ejecutar las actividades de manejo. No así, los escenarios a precio del mercado internacional, donde la TIR presentó un valor bastante menor a la propuesta como tasa ecológica.

Desde una perspectiva internacional el precio al carbono debe situarse como mínimo entre 40 US\$ y 80 US\$ por tonelada para el año 2020, y entre 50 US\$ y 100 US\$ para el año 2030, siempre que exista un entorno normativo favorable (CAPC, 2017). Estos valores permitirían la ejecución de los proyectos en cualquiera de los escenarios propuestos, es decir, existiendo o no la bonificación del Estado, y tasas ecológicas o comerciales.

Cabe destacar las diferencias del comportamiento temporal de los escenarios propuestos, donde el precio de la tonelada de $\mathrm{CO}_{2}$ y la tasa de descuento explican el comportamiento del rendimiento económico de los proyectos. La importancia de la tasa de descuento, la bonificación para el manejo y el horizonte de planificación del proyecto son determinantes para conocer el período de retorno de la inversión. Por ejemplo, en los escenarios analizados, la bonificación al inicio del proyecto podría ser compensada incrementando el precio inicial de la tonelada de $\mathrm{CO}_{2}$ en $\sim 2$ US\$.

En este punto es importante considerar el crecimiento del tipo de bosque en cuestión, ya que su productividad asociada a los mercados de la madera, carbono y miel podría tener cambios significativos en la asignación de precios y subsidios del Estado. Tal es el caso del mercado de carbono, cuyo valor actual en el mercado nacional de 5 US $\$ / t$ determina que los beneficios asociados a bienes derivados de la madera y la producción de miel sean determinantes en la contribución al VAN y a una necesidad de aportes públicos al reconocimiento del capital natural. Por el contrario, un valor de 17,24 US\$/t en el mercado internacional, equivaldría a lo aportado por el valor de mercado conjunto de la madera y la miel.

\section{CONCLUSIONES}

El análisis de este estudio se basó en información real proveniente de 3 ensayos de restauración y una serie de supuestos desarrollados en estudios previos y propuestos por los autores de este trabajo. A partir de los análisis, se puede concluir que la tasa de descuento juega un rol importante en el valor económico de los proyectos. Esto cobra mayor relevancia en iniciativas de restauración cuyos horizontes generalmente son de largo plazo. 
Los escenarios propuestos demuestran la compatibilidad e interdependencias entre la naturaleza ecológica de los bosques y las exigencias directas de los mercados, dando cuenta de la necesidad de incorporar el valor económico de los servicios ecosistémicos en el desarrollo de proyectos de restauración.

Este resultado es novedoso ya que entrega antecedentes que demuestran la importancia de considerar el valor económico de los bienes y servicios ecosistémicos de manera conjunta, lo que permite cambiar y expandir la racionalidad del uso de los bosques como un recurso renovable en relación a la escala temporal asociada al crecimiento de los bosques nativos. De esta forma, el enfoque de la multifuncionalidad de los bosques nativos debe ser incorporado en el desarrollo de políticas públicas, como por ejemplo la Ley de Bosque Nativo, o las decisiones del sector privado, en reconocimiento al valor social de éstos.

El presente trabajo buscó ampliar la visión de manejo tradicional de los bosques abordando la factibilidad de manejarlos con un objetivo multipropósito fomentando el manejo de bosques mixtos siempreverdes. Si bien la información se ajusta a los casos, es importante incluir fuentes de variación asociados a la dinámica de los ecosistemas y los mercados.

Futuros estudios deben enfocarse en comprender el propósito e intereses de los propietarios, el apoyo que puede asumir el Estado en la generación de instrumentos de fomento para corregir las distorsiones de mercado, la importancia de la productividad de los sitios, la calidad de los bosques y las técnicas de manejo ecológicamente sólidas dirigidas hacia la obtención de bosques multifuncionales en cuanto a la provisión conjunta de bienes y servicios.

\section{RECONOCIMIENTOS}

INFOR y los autores agradecen al Fondo de Investigación de la Ley del Bosque Nativo el financiamiento del proyecto 020/2015 "Diversificación de renovales de canelo con pérdida de estructura para el desarrollo hacia bosques mixtos siempreverdes de valor melífero y maderero"; al Ministerio de Agricultura a través de su programa "Restauración de Ecosistemas Forestales Nativos" COD 3031131201; al Instituto de Economía de la Facultad de Ciencias Económicas y Administrativas de la Universidad Austral de Chile y al Centro (CR)2, ANID/FONDAP/15110009.

\section{REFERENCIAS}

Banco Central de Chile, 2020a. Dólar observado. Enero 11, 2020, de Banco Central de Chile En: https://www.bcentral.cl/web/banco-central/inicio. Consulta 12 de enero de 2020.

Banco Central de Chile, 2020b. UTM (ENE). Banco Central de Chile En: https://www.bcentral.cl/web/bancocentral/inicio. Consulta 12 enero 2020.

CAPC, 2017. Informe de la Comisión de Alto Nivel sobre los Precios del Carbono. Comisión de Alto Nivel sobre los Precios del Carbono. Washington, DC, Banco Mundial. Licencia: Creative Commons de Reconocimiento CC BY 3.0 IGO.

Costanza, R.; Arge, R.; Groot, R.; Stephen, F,: Monica, G.; Bruce, H.; Karin, L.; Shahid, N.; Robert, N.; Paruelo, J.; Raskin, R.; Sutton, P. y Belt, M., 1997. The Value of the World's Ecosystem Services and Natural Capital. Nature. 387. 253-260. 10.1016/S0921-8009(98)00020-2.

Donoso, P. y Pilquinao, B., 2013. Desarrollo de sistemas sustentables de manejo multietáneo en bosqus del Tipo Forestal Siempreverde en el centro-sur de Chile. Informe Final Proyecto CONAF 034/2011. En: https://investigacion.conaf.cl/archivos/repositorio_documento/2018/12/034_2011-DOCUMENTOS_INFORMEFINAL.pdf. Consulta 2 de marzo 2020. 
Droppelmann, F.; Grosse, H. y Laroze, A., 2019. Contribución de los bosques nativos y plantados a la mitigación de los impactos del cambio climático en Chile en un contexto de desarrollo sustentable. Ciencia e Investigación Forestal 25(2) 7-35.

EPA, 2016. Technical Update of the Social Cost of Carbon for Regulatory Impact Analysis. Interagency Working Group on Social Cost of Greenhouse Gases, United States Environmental Protection Agency. United States Government.

Figueroa, E. y Pasten, R., 2008. Forest and water: The value of native temperate forests in supplying water for human consumption: A comment. Ecological Economics, 67(2), 153-156. En: https://doi.org/10.1016/j.ecolecon.2008.07.003. Consulta 12 de enero de 2020.

Figueroa, E. y Pasten, R., 2011. Improving benefit transfer for wetland valuation: Income adjustment and economic values of ecosystem goods and services.

Figueroa, E. y Pasten, R., 2015. The economic value of forests in supplying local climate regulation. Aust. J. Agric. Resour. Econ. 59, 446-457. En: https://onlinelibrary.wiley.com/doi/abs/10.1111/1467-8489.12096. Consulta 12 de enero de 2020.

De la Fuente, A. y Pacheco, N., 2017. Biomasa, producción de semillas y fenología de Chusquea montana tras su floración masiva y sincrónica en el Parque Nacional Puyehue, Chile. BOSQUE 38(3): 599-604.

Gayoso, J.; Guerra, J. y Alarcón, D., 2002. Contenido de Carbono y funciones de Biomasa en especies nativas y exóticas. UACh. Informe Técnico Proyecto FONDEF. 157 pp.

Gollier, C., 2010. Ecological discounting. Journal of Economic Theory, 145(2), 812-829.

Hernández, G. y Pinilla, J.C., 2010. Compendio, Propiedades de la Madera de Especies Nativas y Exóticas en Chile. Instituto Forestal. Informe Técnico N 178.

Inflation.eu, 2020. Inflación - información actualizada sobre la inflación. Worldwide Inflation Data. En: https://es.inflation.eu/. Consulta 12 de enero de 2020.

INFOR, 2018. Informe Final Inventario Continuo de Bosques Nativos y Actualización de Plantaciones Forestales. En: https://ifn.infor.cl/index.php/descargas-recursos/descargas/send/2-documentos-inventario-forestal/28-informerecursos-forestal-en-Chile-2018.

IPCC, 2006. Guidelines for National Greenhouse Gas Inventories. Prepared by the National Greenhouse Gas Inventories Programme. Eggleston H.S., Buendia L., Miwa, K. Ngara, T. and Tanabe K. (eds.), Iges, Japón.

Lara A, Soto D, Armesto J, Donoso P, Wernli C, Nahuelhual L, Squeo F., 2003. Componentes científicos clave para una política nacional sobre usos, servicios y conservación de los bosques nativos Chilenos. Libro resultante de la Reunión Científica sobre Bosques Nativos realizada en Valdivia, los días 17-18 de julio de 2003". Universidad Austral de Chile. Iniciativa Científica Milenio de MIDEPLAN. 134

Lara, A.; Urrutia, R.; Little, C. y Martínez, A., 2010. Servicios Ecosistémicos y la Ley del Bosque Nativo: No basta con definirlos. Revista Bosque Nativo 47: 3-9.

Lara, A., Little, C., Nahuelhual, L., Urrutia, R., Díaz, I., 2011. "Lessons, Challenges and Policy Recommendations for the Management, Conservation and Restoration of Native Forests in Chile". En: E. Figueroa (Ed). Successful and Failed Experiences in Biodiversity Conservation: Lessons and Policy Recommendations from the American Continent. Universidad de Chile. Programa de Investigación Domeyco. Santiago, Chile p. 281-327.

Little, C. y Lara, A., 2014. Servicios Ecosistémicos de los Bosques Nativos del Centro Sur de Chile. En: Donoso C., González M.E. y Lara, A. (eds), 2014. Ecología Forestal: Bases para el Manejo Sustentable y Conservación de los Bosques Nativos de Chile. Ediciones Universidad Austral de Chile. 720 p. 
Ley $\mathbf{N}^{\circ} \mathbf{2 0 . 7 8 0 , 2 0 1 4 . ~ R e f o r m a ~ T r i b u t a r i a ~ q u e ~ M o d i f i c a ~ e l ~ S i s t e m a ~ d e ~ T r i b u t a c i o ́ n ~ d e ~ l a ~ R e n t a ~ e ~ I n t r o d u c e ~ D i v e r s o s ~}$ Ajustes en el Sistema Tributario. Ministerio de Hacienda. Diario Oficial de la República de Chile, Santiago, Chile, 29 de Septiembre de 2014.

Ley $\mathbf{N}^{\circ}$ 20.283, 2008. Ley Sobre Recuperación del Bosque Nativo y Fomento Forestal. Ministerio de Agricultura. Diario Oficial de la República de Chile, Santiago, Chile, 30 de julio de 2008.

Manning P, van der Plas F, Soliveres S, Allan E, Maestre FT, Mace G, Whittingham MJ, Fischer M. 2018. Redefining ecosystem multifunctionality. Nat Ecol Evol. 2(3):427-436. DOI: 10.1038/s41559-017-0461-7.

Mascareñas, J., 2008. La Valoración de Proyectos de Inversión Productivos (Project Valuation). Monografías de Juan Mascareñas sobre Finanzas Corporativas, ISSN: 1988-1878, 2008.

Mete, M., 2014. Valor Actual Neto y Tasa de Retorno: Su Utilidad Como Herramientas para el Análisis y Evaluación de Proyectos de Inversión. Fides et Ratio - Revista de Difusió n Cultural y Científica de la Universidad La Salle en Bolivia 7(7): 67-85.

Nahuelhual, L.; Donoso. P.; Lara, A.; Núñez, D.; Oyarzun, C. y Neira, E., 2007. Valuing Ecosystem Services of Chilean Temperate Rainforests. Environment Development and Sustainability 9: 481-499.

Nahuelhual, L. y Núñez, D., 2010. Beneficios Económicos de la Recreación en Áreas Protegidas Públicas del Sur de Chile. Estudios y Perspectivas en Turismo. 19. 703-721.

Ninan, K. y Kontoleon, A., 2016. Valuing forest ecosystem services and disservices - Case study of a protected area in India. Ecosystem Services, Volume 20, Pages 1-14, ISSN 2212-0416, En: https://doi.org/10.1016/j.ecoser.2016.05.001.

Núñez, D.; Nahuelhual, L. y Oyarzún, C., 2006. Forests and Water: The value of forests in providing water for human consumption. Ecological Economics 58: 606-616.

Oyarzún, C. y Huber, A., 1997. Nitrogen export from forested and agricultural watersheds of southern Chile. Gayana Botánica 60(1): 63-68.

Pasten, R.; Olszynski, M. y Hantke-Domas, M., 2018. Does slow and steady win the race? Ecosystem Services in Canadian and Chilean environmental law. Ecosyst. Serv. 29, 240-250. En. https://www.sciencedirect.com/science/article/pii/S2212041616304867. Consulta 12 de enero de 2020.

Pérez, V., 1983. Manual de propiedades físicas y mecánicas de maderas chilenas. Santiago, Chile. Proyecto CONAF/PNUD/FAO-CHI 76/003. Documento de Trabajo N 47. 451 p.

Raychaudhuri, S., 2008. Introduction to Monte Carlo Simulation. In: Proceedings of the 40th Conference on Winter Simulation (WSC '08). Winter Simulation Conference, 91-100.

Saha, S.; Kuene, C. y Bauhus, J., 2016. Lessons Learned from Oak Cluster Planting Trials in Central Europe. Canadian Journal of Forestry Research 47: 139-148.

Vergara, G.; Schlegel, B.; Little, C.; Mujica, R. y Martin, M., 2019. ¿Degradación o Degradado? Necesidad de una Propuesta Conceptual para Recuperar la Funcionalidad y Capacidad Productiva de los Bosques.

Worldbank, 2019a. GDP per capita, PPP (constant 2011 international \$). World Bank International Comparison Program database. En: https://data.worldbank.org/indicator/NY.GDP.PCAP.PP.KD. Consulta 12 de enero de 2020.

Worldbank, 2019b. GDP growth (annual \%) - Chile. World Bank National Accounts Data Files, En: https://data.worldbank.org/indicator/NY.GDP.MKTP.KD.ZG?locations=CL. Consulta 12 de enero de 2020. 